\title{
Correlation of Nutritional Status with Some Micronutrients in Children with Ventricular Septal Defect
}

\author{
Zongyun Xie ${ }^{1}$, Cheng $\mathrm{Xu}^{1}$, Yuxi Zhang ${ }^{1}$, Jing Xu ${ }^{1}$, Yaqin Shu ${ }^{1}$, Jie Zhang ${ }^{2}$ and Xuming $\mathrm{Mo}^{1 *}$ \\ ${ }^{1}$ Department of Cardiothoracic Surgery, Children's Hospital of Nanjing Medical University, Nanjing, 210008, China \\ ${ }^{2}$ Department of Pediatric Surgery, Children's Hospital of Nanjing Medical University, Nanjing, China
}

*Corresponding author: Xuming Mo, Department of Cardiothoracic Surgery, Children's Hospital of Nanjing Medical University, 72 Guangzhou Road, Nanjing, 210008, China

\section{ARTICLE INFO}

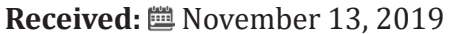

Published: 豐 December 05, 2019

Citation: Zongyun Xie, Cheng Xu, Yuxi Zhang, Jing $\mathrm{Xu}$, Yaqin Shu, Jie Zhang, Xuming Mo. Correlation of Nutritional Status with Some Micronutrients in Children with Ventricular Septal Defect. Biomed J Sci \& Tech Res 23(4)-2019. BJSTR. MS.ID.003925.

Keywords: Ventricular septal defect; Trace elements; Vitamins; Nutritional status

\section{ABSTRACT}

Introduction: The aim of this study was to explore the association between the BMI $\mathrm{Z}$ score and the levels of some selected common nutrients among children under 5 years old with ventricular septal defect (VSD).

Methods: We recruited children under 5 years of age who were diagnosed with VSD from May 2017 to November 2017 at the Children's Hospital of Nanjing Medical University as research subjects to measure their height/length, weight and serum levels of common nutrients. The nutritional status of the selected children was evaluated by the Z-score method.

Results: A total of 146 children under five years old diagnosed with VSD (78 males, 68 females) were recruited. Iron levels were significantly higher among girls than boys. Pearson correlation analysis showed a negative association between VC and the weight for height Z-score (WHZ) ( $\mathrm{r}=-0.172, \mathrm{P}=0.039)$, and there was a positive correlation between VC and the weight for age Z-score (WAZ) $(r=0.247, \mathrm{P}=0.003)$. A comparison of $\mathrm{Z}$ scores $>0$ and $<0$ also revealed that the $\mathrm{VC}$ level in the $\mathrm{WHZ}>0$ group was significantly higher than in the $\mathrm{WHZ}<0$ group $(\mathrm{P}=0.025)$. After linear regression analysis with adjusted as age, gender, and pulmonary artery pressure, there was a positive association between WAZ and VC $(\mathrm{P}=0.035)$.

Conclusion: Our study found that there may be a positive correlation between VC levels and the nutritional status of children with VSD.

Abbreviations: vsd: Ventricular Septal Defect; Whz: Weight for Height Z-Score; Nst: Nutrition Support Team; Haz: Height for Age Z-Score; Waz: Weight for Age Z-Score; Va: Vitamin A; Vb1: Vitamin B1; Vb2: Vitamin B2; Vb6: Vitamin B6; Vb9: Vitamin B9; Vb12: Vitamin B12; Vc: Vitamin C; Vd: Vitamin D; Ve: Vitamin E; Cu: Serum Copper; Zn: Zinc; Ca: Calcium; Mg: Magnesium Fe: Iron; eNOS: Endothelial NO Synthase

\section{Introduction}

Congenital heart disease refers to anatomical abnormalities of the heart and large blood vessels during the embryonic development period or abnormal heart and blood vessel function caused by no closure of the channels that should be closed after birth. Current epidemiological data [1] show that congenital heart disease accounts for approximately $28 \%$ of all major congenital anomalies [2]. On the one hand, due to the progress of society, diagnosis and screening methods have been continuously improving, and thus, increasing numbers of children with congenital heart disease are identified at birth; on the other hand, the process of 
industrialization and urbanization has changed the environment. Increasing numbers of women have postponed childbearing age, especially in developed countries, leading to a high incidence of congenital abnormalities. The incidence of congenital heart disease is increasing each year, so studies of congenital heart disease are receiving increased attention.

Due to the abnormal hemodynamics caused by congenital heart disease, children with congenital heart disease often suffer from hypoxia, hyperthyroidism, frequent respiratory infections, and poor gastrointestinal absorption [3], and growth retardation and malnutrition are common in children with congenital heart disease. At present, the European Society of Pediatric Gastrointestinal Hepatology Nutrition has called for the establishment of a "nutrition support team (NST)" aimed at scientifically effective nutritional management of hospitalized children and reduction of the prevalence of malnutrition [4]. However, with the trend in younger children with congenital heart disease becoming increasingly obvious [1], the earlier the time for surgery is, the more favorable it is for children to catch up with children of the same age earlier in their growth and development [5]. Therefore, we should focus our attention and intervention on the nutritional status of children with congenital heart disease.

However, clinical interventions for nutrition are mostly focused on carbohydrates and fats that can provide actual calories, and the importance of micronutrients is often overlooked. Although humans need a few vitamins and trace elements, they are the trace organics necessary for maintaining body health. These substances neither provide energy sources in the body nor constitute the raw material of body tissues or participate in the formation of human cells; however, these substances play a crucial role in the growth, metabolism, and development of the human body. Due to a lack of attention to dietary supplements and poor digestive functions such as malabsorption and diarrhea, children with congenital heart disease are at risk of these micronutrient deficiencies. In addition, diuretics are used in children with congenital heart disease. Diuretics excrete water and sodium through the kidneys to improve circulatory function and at the same time, excrete other watersoluble molecules, including potassium, magnesium, and vitamin $\mathrm{C}$ [6]. Studies have shown that early identification of abnormalities in micronutrients and immunodeficiency can help reduce the incidence in children after cardiac surgery [7].

It is necessary to carry out studies of the preoperative nutritional status of children with congenital heart disease, especially infants and young children, and investigate the correlations between nutritional status and various common trace elements and vitamins in our country. Studies have shown that the nutritional status of children with cyanotic and noncyanotic congenital heart disease is significantly different: children with noncyanotic congenital heart disease are more prone to malnutrition [8], and the nutritional status of these children and its influencing factors deserve more attention. Ventricular septal defect is the representative disease type in noncyanotic congenital heart disease. It is the most common clinical congenital heart disease, accounting for approximately $20 \%$ of congenital heart disease [9]. Therefore, in order to ensure the representativeness of the research sample and effectively control for confounding, this study selected ventricular septal defect as the research disease. This research will study the preoperative nutritional status, common elements, and vitamin levels of children with ventricular septal defect younger than 5 years of age and explore the relevance between them, so that we can provide preventive measures for preoperative malnutrition and guide postoperative nutritional intervention.

\section{Methods}

\section{Population Included}

A cross-sectional study was conducted at the Department of Cardiothoracic Surgery of Children's Hospital of Nanjing Medical University from May 2017 to October 2017. This study comprised 146 children younger than 5 years who were diagnosed with ventricular septal defects by echocardiography and excluded children with other congenital malformations or other diseases (including genetic diseases) affecting growth or nutritional status. The study was approved by the Ethical Committee of Children's Hospital of Nanjing Medical University, and informed consent was obtained from the guardian of the children.

\section{Nutrition Evaluation Method}

Anthropometric measurements included weight (kilograms) and length (centimeters), and they were performed according to standard World Health Organization (WHO) procedures [10]. The Z-score method provided by the National Health Statistics Center and recommended by the WHO was used as the evaluation standard. The Z-score was calculated according to the formula Z-score=(measurement value-median value of the same-age and same-sex reference standard)/standard deviation of the reference standard, including height for age Z-score (HAZ), weight for age Z-score (WAZ), and weight for height Z-score (WHZ). The latest WHO reference standard in 2006 was selected to calculate the $\mathrm{Z}$ value [11].

\section{Vitamins and Elements Measurement}

Serum levels of vitamin A (VA), vitamin B1 (VB1), vitamin B2 (VB2), vitamin B6 (VB6), vitamin B9 (VB9), vitamin B12 (VB12), vitamin $C$ (VC), vitamin D (VD), and vitamin E (VE) were measured by a BH5100T Whole Blood Multielement Analyzer. Serum copper $(\mathrm{Cu})$, zinc $(\mathrm{Zn})$, calcium $(\mathrm{Ca})$, magnesium $(\mathrm{Mg})$ and iron $(\mathrm{Fe})$ levels were measured by an LK3000V vitamin detector (Tianjin Lanbiao Electronic Technology Development Co., Ltd.).

\section{Data Analysis}

In this study, SPSS 23.0 statistical software was used for statistical analysis, and $\mathrm{P}<0.05$ indicates a significant difference. General information, zinc, iron, magnesium, copper, calcium, VA, 
VB1, VB2, VB6, VB9, VB12, VC, VE, and VD levels of male and female children are described with the mean plus standard deviation. Mann-Whitney $U$ tests verified whether there was a significant difference between different genders. Pearson bivariate correlation analysis was used to evaluate the correlation between $\mathrm{Z}$ scores (e.g., WHZ, WAZ, HAZ) and Zn, Fe, Mg, Cu, Ca, VA, VB1, VB2, VB6, VB9, VB12, VC, VE, and VD. Z-values WHZ, WAZ, and HAZ were grouped by 0 , and we compared the levels of $\mathrm{Zn}, \mathrm{Fe}, \mathrm{Mg}, \mathrm{Cu}, \mathrm{Ca}$, VA, VB1, VB2, VB6, VB9, VB12, VC, VE, and VD between groups, verified whether they met normality and used an independent t-test for data that satisfied normality. If the data did not satisfy normality, the MannWhitney $U$ test was used. Using logistic regression analysis, the confidence interval level was 95\%. After correction for age, gender, and pulmonary artery pressure, the ratios of Z-values WHZ, WAZ, HAZ and zinc, iron, magnesium, copper, calcium, VA, VB1, VB2, VB6, VB9, VB12, VC, VE, and VD were analyzed, and the 95\% confidence interval was calculated.

\section{Results}

\section{Demographic Characteristics of the Participants}

There were 68 females and 78 males; for females, the mean age was $18.75 \pm 17.75$ months, mean weight was $9.97 \pm 5.15 \mathrm{~kg}$, and mean height was $78.16 \pm 18.33 \mathrm{~cm}$, and for males, the mean age was $21.33 \pm 18.56$ months, mean weight was $11.13 \pm 5.20 \mathrm{~kg}$, and mean height was $81.46 \pm 18.07 \mathrm{~cm}$. The iron levels of females were significantly higher than those of males (male vs female: iron, $415.03 \pm 33.80$ vs $427.87 \pm 38.33$, $\mathrm{P}=0.033$ ) (Table 1 ). However, no statistically significant differences of other vitamins and trace elements were existing in males and females.

Table 1: General situation between different genders.

\begin{tabular}{|c|c|c|c|}
\hline & Boy $(\mathbf{N}=78)$ & Girl $\mathbf{( N = 6 8 )}$ & P value \\
\hline Age (month) & $21.33 \pm 18.56$ & $18.75 \pm 17.75$ & 0.373 \\
\hline Weigh (Kg) & $11.13 \pm 5.20$ & $9.97 \pm 5.15$ & 0.116 \\
\hline Height(cm) & $81.46 \pm 18.07$ & $78.16 \pm 18.33$ & 0.199 \\
\hline $\mathrm{Zn}$ & $4.12 \pm 0.92$ & $4.13 \pm 0.84$ & 0.803 \\
\hline $\mathrm{Fe}$ & $415.03 \pm 33.80$ & $427.87 \pm 38.33$ & 0.033 \\
\hline $\mathrm{Mg}$ & $35.13 \pm 5.78$ & $35.61 \pm 5.60$ & 0.558 \\
\hline $\mathrm{Cu}$ & $1.38 \pm 0.38$ & $1.36 \pm 0.46$ & 0.623 \\
\hline $\mathrm{Ca}$ & $75.50 \pm 6.20$ & $75.23 \pm 6.85$ & 0.749 \\
\hline $\mathrm{VA}$ & $0.63 \pm 0.36$ & $0.64 \pm 0.29$ & 0.376 \\
\hline
\end{tabular}

\begin{tabular}{|c|c|c|c|}
\hline VB1 & $49.23 \pm 15.39$ & $50.69 \pm 13.93$ & 0.366 \\
\hline VB2 & $526.73 \pm 189.25$ & $481.61 \pm 225.51$ & 0.181 \\
\hline VB6 & $20.70 \pm 9.62$ & $19.90 \pm 7.91$ & 0.984 \\
\hline VB9 & $10.56 \pm 5.23$ & $9.82 \pm 4.18$ & 0.531 \\
\hline VB12 & $387.09 \pm 218.60$ & $383.42 \pm 210.85$ & 0.899 \\
\hline VC & $33.21 \pm 8.30$ & $32.03 \pm 8.68$ & 0.323 \\
\hline VE & $11.38 \pm 1.03$ & $11.37 \pm 1.22$ & 0.727 \\
\hline VD & $81.32 \pm 24.22$ & $73.47 \pm 26.11$ & 0.196 \\
\hline WHZ & $-0.34 \pm 1.65$ & $-0.64 \pm 1.80$ & 0.425 \\
\hline WAZ & $-0.32 \pm 1.44$ & $-0.65 \pm 1.66$ & 0.280 \\
\hline HAZ & $-0.01 \pm 1.83$ & $0.22 \pm 1.55$ & 0.502 \\
\hline
\end{tabular}

\section{Correlation Analysis of Elements and Vitamins and $\mathrm{Z}$}

\section{Scores}

Pearson correlation analysis (Table 2) showed that there was a significant correlation between VC and WHZ ( $\mathrm{r}=-0.172, \mathrm{P}=0.039$ ), and there was also a significant correlation between $\mathrm{VC}$ and $\mathrm{WAZ}$ ( $\mathrm{r}=0.247, \mathrm{P}=0.003)$. While, no significant correlation was found between $\mathrm{Zn}, \mathrm{Fe}, \mathrm{Mg}, \mathrm{Cu}, \mathrm{Ca}$ levels and the Z-value scores WHZ, WAZ, and HAZ. A comparison of Z scores $>0$ and $<0$ showed that VC levels in the WHZ $>0$ group were significantly higher than those in the $\mathrm{WHZ}<0$ group $(\mathrm{P}=0.025)$, as shown in (Table 3 ). As a result, we conducted a linear regression after adjusting gender and age and found VC was positively associated with between WAZ. The P value was 0.035 , as shown in (Figure 1) and (Table 4).

Table 2: Pearson correlation analysis of some common vitamins and $\mathrm{Z}$ scores.

\begin{tabular}{|c|c|c|c|c|c|c|}
\hline & WHZ & & WAZ & & HAZ & \\
\hline VA & -0.070 & 0.402 & 0.024 & 0.779 & 0.062 & 0.457 \\
\hline VB1 & -0.012 & 0.884 & -0.012 & 0.888 & -0.009 & 0.917 \\
\hline VB2 & 0.009 & 0.919 & -0.031 & 0.714 & -0.052 & 0.539 \\
\hline VB6 & -0.049 & 0.562 & 0.029 & 0.734 & 0.077 & 0.360 \\
\hline VB9 & -0.27 & 0.750 & 0.048 & 0.569 & 0.077 & 0.361 \\
\hline VB12 & 0.082 & 0.330 & 0.078 & 0.350 & 0.001 & 0.992 \\
\hline VC & 0.172 & 0.039 & 0.247 & 0.003 & 0.146 & 0.081 \\
\hline VE & -0.152 & 0.070 & -0.077 & 0.360 & 0.030 & 0.723 \\
\hline VD & 0.111 & 0.186 & 0.154 & 0.066 & 0.010 & 0.902 \\
\hline
\end{tabular}

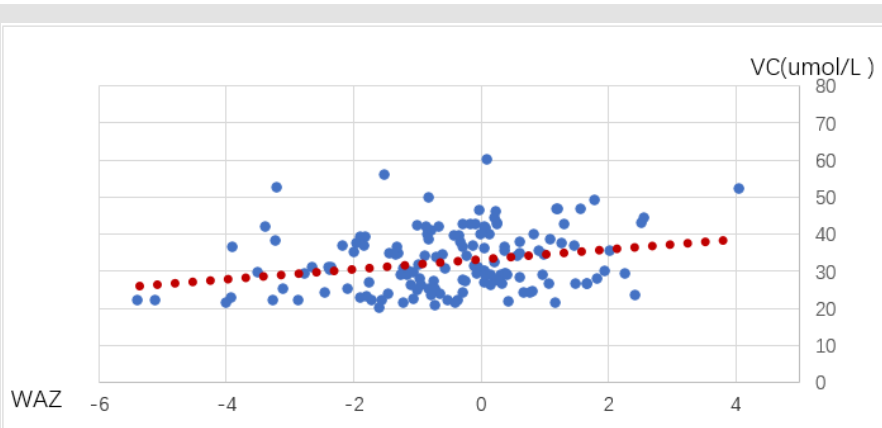

Figure 1: A scatter plot and a fitted line with 95\% CI of the relationship between vitamin C and weight for age Z-score in children with ventricular septal defect. 
Table 3: Comparative analysis of partial vitamin levels of $Z$ score $>0$ group and $Z$ score $<0$ group.

\begin{tabular}{|c|c|c|c|c|c|c|c|c|c|}
\hline & \multicolumn{3}{|c}{ WHZ } & \multicolumn{3}{c|}{ WAZ } & \multicolumn{3}{c|}{ HAZ } \\
\hline & $<\mathbf{0}$ & $>\mathbf{0}$ & P value & $<\mathbf{0}$ & $>\mathbf{0}$ & P value & $<0$ & $>\mathbf{0}$ & P value \\
\hline VA & $0.7 \pm 0.4$ & $0.6 \pm 0.3$ & 0.561 & $0.7 \pm 0.3$ & $0.6 \pm 0.3$ & 0.705 & $0.6 \pm 0.3$ & $0.7 \pm 0.4$ & 0.221 \\
\hline VB1 & $50.6 \pm 15.9$ & $48.8 \pm 12.8$ & 0.522 & $50.1 \pm 16.0$ & $49.5 \pm 12.7$ & 0.968 & $50.9 \pm 15.4$ & $48.8 \pm 14.3$ & 0.680 \\
\hline VB2 & $491.3 \pm 215.8$ & $531.2 \pm 192.4$ & 0.321 & $510.6 \pm 219.0$ & $503.2 \pm 189.0$ & 0.835 & $506.1 \pm 207.2$ & $509.3 \pm 207.9$ & 0.804 \\
\hline VB6 & $20.3 \pm 9.0$ & $20.5 \pm 8.7$ & 0.596 & $20.3 \pm 9.5$ & $20.4 \pm 7.9$ & 0.397 & $20.8 \pm 9.7$ & $20.0 \pm 7.9$ & 0.851 \\
\hline VB9 & $10.4 \pm 5.42$ & $10.0 \pm 3.7$ & 0.722 & $10.1 \pm 5.2$ & $10.4 \pm 4.14$ & 0.343 & $9.8 \pm 3.8$ & $10.7 \pm 5.6$ & 0.686 \\
\hline VB12 & $371.4 \pm 212.5$ & $397.3 \pm 209.2$ & 0.397 & $383.3 \pm 214.1$ & $379.7 \pm 207.7$ & 0.977 & $379.6 \pm 212.4$ & $384.4 \pm 210.6$ & 0.870 \\
\hline VC & $31.4 \pm 8.7$ & $34.1 \pm 7.7$ & 0.025 & $31.4 \pm 8.1$ & $34.2 \pm 8.5$ & 0.052 & $31.6 \pm 7.7$ & $33.5 \pm 8.77$ & 0.180 \\
\hline VE & $11.5 \pm 1.3$ & $11.2 \pm 0.9$ & 0.554 & $11.5 \pm 1.3$ & $11.2 \pm 0.9$ & 0.325 & $11.4 \pm 1.2$ & $11.3 \pm 0.99$ & 0.826 \\
\hline VD & $75.3 \pm 25.9$ & $80.8 \pm 24.5$ & 0.101 & $76.2 \pm 26.7$ & $79.6 \pm 23.4$ & 0.288 & $78.8 \pm 25.3$ & $76.2 \pm 25.6$ & 0.633 \\
\hline
\end{tabular}

Table 4: Partial vitamin and $\mathrm{Z}$ value regression analysis.

\begin{tabular}{|c|c|c|c|c|c|c|}
\hline & WHZ & & WAZ & & HAZ & Beta 95\%CIs \\
\hline & Beta (95\%CIs) & P value & Beta 95\%CIs & P value & P value \\
\hline VA & $-0.31(-1.05,0.44)$ & 0.417 & $0.08(-0.53,0.70)$ & 0.788 & $0.24(-0.62,1.09)$ & 0.587 \\
\hline VB1 & $-0.01(-0.02,0.01)$ & 0.570 & $-0.01(-0.02,0.00)$ & 0.463 & $-0.01(-0.02,0.12)$ & 0.780 \\
\hline VB2 & $0.00(-0.01,0.01)$ & 0.586 & $-0.01(-0.01,0.00)$ & 0.195 & $-0.01(-0.01,0.00)$ & 0.383 \\
\hline VB6 & $0.01(-0.02,0.04)$ & 0.663 & $0.01(-0.02,0.03)$ & 0.597 & $-0.01(-0.03,0.02)$ & 0.427 \\
\hline VB9 & $-0.03(-0.08,0.02)$ & 0.233 & $-0.01(-0.05,0.04)$ & 0.750 & $0.02(-0.04,0.08)$ & 0.553 \\
\hline VB12 & $0.01(-0.01,0.01)$ & 0.370 & $0.00(-0.01,0.01)$ & 0.622 & $0.00(-0.01,0.01)$ & 0.650 \\
\hline VC & $0.02(-0.01,0.05)$ & 0.245 & $0.03(0.00,0.05)$ & 0.035 & $0.02(-0.01,0.05)$ & 0.243 \\
\hline VE & $-0.08(-0.30,0.14)$ & 0.469 & $0.05(-0.14,0.22)$ & 0.625 & $0.10(-0.15,0.35)$ & 0.434 \\
\hline VD & $-0.01(-0.02,0.00)$ & 0.272 & $0.00(-0.01,0.01)$ & 0.460 & $0.00(-0.02,-0.01)$ & 0.462 \\
\hline
\end{tabular}

Substances are Log10

Corrected gender, age, pulmonary artery pressure.

\section{Discussion}

The results of our study revealed a linear regression relationship between VC and WAZ, with a P value of 0.035 , suggesting a possible correlation between VC and the nutritional status of children with congenital heart disease. In people with malnutrition caused by chronic diseases, poor eating habits, malabsorption, and other factors, obvious VC deficiency can often be seen [12]. Some animal experiments have shown that VC is an important vitamin for maintaining normal growth and physiological function [13], playing an important role in the growth process [9], collagen formation [14, 15], iron metabolism and hematology [9], reproduction [16], stress response [17], and wound healing [18]. Wang et al. [19]. found that dietary supplementation with VC can significantly increase the daily weight of juvenile ducks. A study by Zhou et al. [20] also showed that increases in body weight, specific growth ratio, protein efficiency ratio, and feed efficiency of VC-deficient fish were lower than those of fish supplemented with VC. In addition, growth performance, antioxidant capacity, and humoral immune parameters of juvenile cobia supplemented with VC were significantly improved $[19,20]$.

Heart failure increased pulmonary arterial pressure, and anemia are risk factors for protein energy malnutrition in children with congenital heart disease [9], and some physiological functions of VC can alleviate these risk factors. A considerable amount of research is currently focused on the use of VC to prevent heart failure [21], mainly because of its ability to prevent oxidative damage. Reactive oxygen or nitrogen species (ROS/RNS) are the most common oxidants in the human body and play a key role in aggravating human heart failure [9]. Studies have shown that ROS and RNS can change the properties of lipids, proteins, and nucleic acids, leading to myocardial dysfunction, and myocardial oxidative damage is closely related to congestive heart failure [22]. On the other hand, ascorbate regulates vasodilation by increasing NO synthesis and improving bioavailability [23]. Endothelial NO synthase (eNOS) produces NO, which then diffuses into the smooth muscle cell layer of the vascular wall and causes vasodilation through interaction with soluble guanylate cyclase. Another clinical application of VC is to increase the absorption of nonheme iron [12]. In the small intestine, VC can effectively increase dietary iron transport in intestinal epithelial cells. When anemia patients are supplemented with iron and VC, the synthesis of hemoglobin can be promoted. In addition, studies have also shown [24] that a longacting VC derivative called ascorbic acid 2-phosphate stimulates cell growth and increases mRNA type III collagen in human osteogenic MG-63 cells and normal human osteoblasts, as well as expression in human bone marrow mesenchymal stem cells, thereby stimulating 
proliferation and differentiation of human osteoblast-like cells. In summary, we speculate that VC can indirectly influence the nutritional and growth status of children with congenital heart disease by exerting its biological functions.

VC is mainly related to WAZ, which can reflect acute malnutrition in patients, and we have previously speculated that some trace elements may be related to HAZ, reflecting chronic malnutrition in patients. However, in our research, no correlation was found between $\mathrm{Cu}, \mathrm{Fe}, \mathrm{Zn}, \mathrm{Mg}$, Ca levels and WHZ, WAZ, HAZ; the reasons need further research. Our findings suggest that there is a correlation between VC and WAZ. WAZ is an indicator of acute malnutrition, reflecting the problem of malnutrition at this stage. It is more marked by factors such as disease, feeding method, food choice, and poor nutritional habits [25]. It can be speculated that supplementation with VC can alleviate acute nutritional problems soon.

\section{Conclusion}

This study revealed a positive correlation between VC and the nutritional status of children with ventricular septal defects. Further, our results may have implications from the perspective of VC on the nutritional status of children with clinical congenital heart disease intervention, to prevent malnutrition in children before surgery, while guiding clinical staff to carry out postoperative nutritional support in children with congenital heart disease.

\section{Acknowledgement}

This work was supported by funding from The National Key Research and Development Program of China (2017YFC1308105, 2016YFC1101001), the Maternal and Child Health Research Project of Jiangsu Province (F201755), the China Postdoctoral Science Foundation (2018M630585), the Key Project of Science and Technology Development Fund of Nanjing Medical University (2017NJMUZD060, 2017NJMU067), Nanjing Medical University School Project (NMUC2018012A), and Key Project supported by Medical Science and technology development Foundation, Nanjing Department of Health (YKK18139, 201723008).

\section{Competing Interests}

The authors declare that they have no competing interests.

\section{Compliance with Ethical Standards}

\section{Conflict of Interest}

The authors have no conflicts of interest to declare.

\section{Ethical Approval}

All procedures performed in study were in accordance with the ethical standards of national research committee and with the 1964 Helsinki Declaration and its later amendments.

\section{Informed Consent}

Informed consent was obtained from all guardians of patients included in the study.

\section{References}

1. van der Linde D, Konings EE, Slager MA, Witsenburg M, Helbing WA, et al. (2011) Birth prevalence of congenital heart disease worldwide: a systematic review and meta-analysis. J Am Coll Cardiol 58(21): 22412247.

2. Dolk H (2011) Congenital heart defects in Europe: prevalence and perinatal mortality 2000 to 2005. Circulation 123(8): 841-849.

3. Schwalbe Terilli CR, Hartman DH, Nagle ML, Gallagher PR, Ittenbach RF, et al. (2009) Enteral feeding and caloric intake in neonates after cardiac surgery. Am J Crit Care 18(1): 52-57.

4. Agostoni C, Irene Axelson, Virginie Colomb, Olivier Goulet, Berthold Koletzko, et al. (2005) The need for nutrition support teams in pediatric units: a commentary by the ESPGHAN committee on nutrition. J Pediatr Gastroenterol Nutr 41(1): 8-11.

5. Steltzer M, N Rudd, B Pick (2005) Nutrition care for newborns with congenital heart disease. Clin Perinatol 32(4): 1017-1030.

6. Sica DA (2007) Loop diuretic therapy thiamine balance and heart failure. Congest Heart Fail 13(4): 244-247.

7. Cabrera AG, P Prodhan, AT Bhutta (2010) Nutritional challenges and outcomes after surgery for congenital heart disease. Curr Opin Cardiol 25(2): 88-94.

8. Varan B, K Tokel, G Yilmaz (1999) Malnutrition and growth failure in cyanotic and acyanotic congenital heart disease with and without pulmonary hypertension. Arch Dis Child 81(1): 49-52.

9. Leirgul E, Fomina T, Brodwall K, Greve G, Holmstrøm H, et al. (2014) Birth prevalence of congenital heart defects in Norway 1994-2009--a nationwide study. Am Heart J 168(6): 956-964.

10. (1995) Physical status: the use and interpretation of anthropometry. Report of a WHO Expert Committee. World Health Organ Tech Rep Ser 854: 1-452.

11. Ratanachu Ek S, A Pongdara (2011) Nutritional status of pediatric patients with congenital heart disease: pre- and post-cardiac surgery. J Med Assoc Thai 94 Suppl 3: 133-137.

12. Lykkesfeldt J, AJ Michels, B Frei (2014) Vitamin C. Adv Nutr 5(1): 16-18.

13. Kumari J, PK Sahoo (2005) High dietary vitamin C affects growth nonspecific immune responses and disease resistance in Asian catfish Clarias batrachus. Mol Cell Biochem 280(1-2): 25-33.

14. (1982) Effect of dietary ascorbic acid levels on collagen formation in rainbow trout [Salmo gairdnerii]. 4: 553-557.

15. Sandell L JD, Jon C (1988) Effects of Ascorbic Acid on Collagen Mrna Levels in Short Term Chondrocyte Cultures. Journal Connective Tissue Research 17(1): 11-23.

16. Soliman Ak, Kroberts R J (1986) The effect of varying forms of dietary ascorbic acid on the nutrition of juvenile tilapias (Oreochromis niloticus). 52(1): 1-11.

17. Wedemeyer G (1969) Stress-induced ascorbic acid depletion and cortisol production in two salmonid fishes. 29(3): 1247-1252.

18. Gabaudan, JVV Critical review of the requirements of ascorbic acid in cold and cool water fishes (salmonids, percids, plecoglossids, and flatfishes)Aquatic organisms and practical aquaculture. p. 33-49.

19. WangA, Xie F, Wang YH, Wu JL (2011) Effects of vitamin C supplementation on growth performance and antioxidant status of layer ducklings. J Anim Physiol Anim Nutr (Berl) 95(4): 533-540.

20.Zhou Qa, Ligai Wang, Hualang Wang, Fengjun Xie, Tuo Wang, et al. (2012) Effect of dietary vitamin $\mathrm{C}$ on the growth performance and innate immunity of juvenile cobia (Rachycentron canadum). Fish \& Shellfish Immunology 32(6): 969-976.

21. Nain S, Wojnarowicz C (2008) Effects of dietary vitamin E and C supplementation on heart failure in fast growing commercial broiler chickens. Br Poult Sci 49(6): 697-704. 
22. Orrenius s, Vladimir, zhivotovsky Boris (2007) Mitochondrial oxidative stress : Implications for cell death. Annu Rev Pharmacol Toxicol 47: 143184.

23. Tveden-Nyborg P, Jens (2013) Does vitamin C deficiency increase lifestyle-associated vascular disease progression? Evidence based on experimental and clinical studies. Antioxid Redox Signal 19(17): 20842105

\section{ISSN: 2574-1241}

DOI: 10.26717/BJSTR.2019.23.003925

Xuming Mo. Biomed J Sci \& Tech Res

(C) (P) This work is licensed under Creative

Submission Link: https://biomedres.us/submit-manuscript.php
24. Maehata Y, Takamizawa Shinji, Ozawa Shigeyuki, Izukuri Kazuhito, Kato Yasumasa, et al. (2007) Type III collagen is essential for growth acceleration of human osteoblastic cells by ascorbic acid 2-phosphate, a long-acting vitamin C derivative. Matrix Biol 26(5): 371-382.

25. (2014) Organic light-emitting diode display device having deep and skyblue sub-pixels.

BIOMEDICAL
RESEARCHES $\quad \begin{aligned} & \text { Assets of Publishing with us } \\ & \text { - Global archiving of articles }\end{aligned}$

